\title{
Cirurgia de Revascularização Miocárdica no Diabetes Mellitus
}

\begin{abstract}
RESUMO
Diabetes mellitus está presente em 25 a $30 \%$ de pacientes com doença arterial coronariana que necessitam cirurgia. Os pacientes diabéticos têm prognóstico distinto dos não-diabéticos, tanto na evolução clínica como nos resultados cirúrgicos. A cirurgia de revascularização está indicada em lesões de 2 ou mais coronárias, nos diabéticos. Porém, pode ser preferida, em relação à intervenção percutânea, em lesões de 1 artéria, quando esta for a descendente anterior ou houver área extensa sob isquemia. Diabéticos candidatos a transplante renal devem ser submetidos à cinecoronariografia e, se necessário, à revascularização. Pacientes diabéticos apresentam maior morbidade, como internação prolongada, infecções, insuficiência respiratória, complicações renais e cerebrais. No entanto, não há maior mortalidade, exceto nos pacientes sem diagnóstico prévio. $\mathrm{Na}$ evolução tardia, diabetes representa fator de risco para oclusões de enxertos e mortalidade, tanto de causa cardíaca como de outras causas. Embora os riscos sejam maiores, a cirurgia de revascularização resulta em melhor qualidade de vida e sobrevida em relação ao tratamento clínico e à angioplastia percutânea, especialmente nos usuários de insulina ou com enxertos de artérias mamárias. (Arq Bras Endocrinol Metab 2007; 51/2:345-351)
\end{abstract}

Descritores: Diabetes mellitus; Aterosclerose coronariana; Revascularização miocárdica

\begin{abstract}
Coronary Artery Surgery in Patients with Diabetes Mellitus.

Diabetes mellitus is present in $25-30 \%$ of patients undergoing coronary artery bypass grafts surgery. Early and late post-operative prognoses are different for the diabetic patient. Coronary artery bypass grafts are indicated in 2 or more vessel lesions, but it can also be preferred to percutaneous angioplasty in 1-vessel lesions, when this is the anterior descending artery or there is a great area under ischemia. Diabetic candidates to renal transplant must be investigated and revascularized pre-operatively, if necessary. Morbidity is greater in these patients, mainly due to respiratory, renal and cerebral complications and wound infections. Intensive care unit and hospital length of stay are more prolonged, but there is not increased early mortality. Diabetes mellitus represents an independent risk factor for late graft failure and mortality from cardiac and general causes. Although under an increased risk, coronary artery surgery results in better quality of life and late survival in the diabetic patients with severe coronary artery disease, as compared to medical treatment and percutaneous coronary angioplasty, specially in those who use insulin and when internal thoracic arterial grafts are implanted. (Arq Bras Endocrinol Metab 2007;51/2:345-351)
\end{abstract}

Keywords: Diabetes mellitus; Coronary artery bypass grafts; Coronary atherosclerosis revisão

RENATO A.K. KALIL

Instituto de Cardiologia do Rio

Grande do Sul / Fundação

Universitária de Cardiologia

(IC/FUC), Porto Alegre, RS.
Recebido em 18/17/06

Aceito em 23/17/06 
$\mathrm{O}$ DESENVOLVIMENTO DA CINECORONARIOGRAFIA por Sones e Shirey na Cleveland Clinic, na década de 1960, tornou possível a identificação de lesões ateroscleróticas obstrutivas nas artérias coronárias e o planejamento de intervenções cirúrgicas, bem como, mais tarde, as intervenções por cateter propostas por Gruntzig. Tentativas anteriores de revascularização baseavam-se em métodos cegos para a anatomia das artérias coronárias. Vineberg e Miller, de Montreal, relataram o implante direto da artéria mamária interna, com seus ramos sangrantes, no interior do miocárdio, para prover e manter circulação colateral, método que mostrou certa eficácia. Em 1954, Murray e cols. relataram estudos experimentais de anastomose direta da artéria mamária para artérias coronárias. Logo a seguir, Longmire descreveu uma série de casos de endarterectomia coronariana sem circulação extra-corpórea. Após o advento deste recurso para facilitar o procedimento, Senning relatou tratamento de estenose coronariana por arterioplastia com enxertos. Embora tenha havido pouca divulgação na época, a primeira cirurgia de anastomose da artéria mamária na artéria coronária descendente anterior foi realizada por Kolesov, em Leningrado, em 1964. A partir de 1967, Effler e Favaloro, ainda na Cleveland Clinic, começaram os esforços pioneiros de revascularização com enxertos de veia safena, baseados nas lesões demonstradas pelas cinecoronariografias de Sones (1). A partir de então, o progresso destes procedimentos se espalhou rapidamente pelo mundo inteiro, apesar de forte e intensa resistência de cardiologistas mais conservadores, terminando por se impor como terapêutica eficaz no alívio dos sintomas, na prevenção de infartos do miocárdio e no aumento da longevidade.

A cirurgia de revascularização direta do miocárdio foi um dos procedimentos mais testados e contestados, se não o mais contestado, entre os procedimentos médicos, apesar da lógica da sua proposta e das evidências de eficácia, necessitando muitos anos de discussão para ser finalmente aceito de forma universal. No Brasil, foi introduzida no início dos anos 1970, em São Paulo, pelas equipes dirigidas por E.J. Zerbini e por Adib Jatene, e logo a seguir no Rio Grande do Sul, pela equipe chefiada por Ivo Nesralla, no Instituto de Cardiologia do Rio Grande do Sul.

A prevalência de diabetes mellitus (DM) em todo o mundo ocidental vem aumentando em níveis alarmantes nos últimos anos, tendo sido encontrado aumento de $8,9 \%$, em 1992, para 12,4\% em 2000, em nosso meio (2). A doença arterial coronariana está freqüentemente a ela associada. Os pacientes diabéticos têm prognóstico distinto dos não-diabéticos em relação à doença coronariana, bem como evoluções distintas quando tratados por intervenção percutânea com cateter ou por cirurgia (3). Estudos recentes confirmam informações anteriores de que a presença de diabetes mellitus é fator de risco independente para mortalidade tardia pós-operatória de cirurgia de revascularização miocárdica, com razão de chances de 1,73 para óbitos de causa cardíaca (4) e de 2,94 para mortalidade global, de todas as causas (5).

A maior parte das informações que embasam as indicações de revascularização percutânea ou cirúrgica provém do estudo BARI, referido adiante, publicado em 1997, além de alguns estudos posteriores. Revisão atualizada do tema de revascularização miocárdica nos pacientes com cardiopatia isquêmica e diabetes foi recentemente publicada por Quadros e cols. (6).

As evidências disponíveis levam à tendência maior de indicação de cirurgia direta de revascularização no diabético com lesão multiarterial. Entretanto, um consenso amplo não foi alcançado, porque os achados não estão uniformemente confirmados por registros e outros estudos. Com a recente introdução de stents farmacológicos, que prometem índices de reestenose menores, há expectativa quanto à melhoria do prognóstico de pacientes submetidos à intervenção percutânea. Os stents farmacológicos estão sendo estudados em ensaios clínicos a serem publicados nos próximos anos, e seu desempenho tardio ainda é desconhecido. Neste artigo, abordaremos especificamente os fenômenos relacionados à cirurgia de revascularização direta com enxertos de veia safena e de artéria mamária interna.

$\mathrm{Na}$ avaliação do paciente para indicação cirúrgica e na análise de resultados, deve-se ter em mente que a doença arterial coronariana é a principal causa de óbito em pacientes diabéticos e está relacionada à mortalidade 3 vezes maior nestes pacientes em relação aos não-diabéticos (7). No diabético, não apenas a incidência de infarto do miocárdio é maior, como o seu tratamento é mais complexo e o prognóstico mais comprometido, quanto à mortalidade e complicações cardiovasculares, que no paciente não-diabético (8).

A alternativa de revascularização cirúrgica do miocárdio, com implantes de pontes de safena ou de artéria mamária interna, deve ser considerada em vista dos benefícios clínicos em médio e longo prazos e das limitações do tratamento medicamentoso e da intervenção percutânea neste grupo de pacientes. Ao se indicar cirurgia na presença de diabetes mellitus, devemos considerar a aceitação de potencial maior risco cirúrgico e de cuidados especiais no manejo perioperatório. 


\section{INDICAÇÃO CIRÚRGICA}

Ressalvadas as peculiaridades do paciente com DM, a indicação de cirurgia de revascularização segue os princípios recomendados para as síndromes anginosas e doença arterial coronariana expostos nas diretrizes da American Heart Association/American College of Cardiology (8) e, em nosso meio, da Sociedade Brasileira de Cardiologia $(3,9)$ (tabelas 1, 2 e 3).

Deve ser considerado que os pacientes diabéticos têm prognóstico diferente dos não-diabéticos quanto à cirurgia de revascularização, conforme exposto nas demais seç̧ões deste artigo. Embora as intervenções percutâneas sejam muitas vezes preferidas e realizadas nos pacientes com lesões uniarteriais, deve ser ressaltado que os diabéticos apresentam maior taxa de reestenose, e este fato deve ser tomado em consideração no momento da indicação. Em lesões da artéria descendente anterior ou quando há grande área isquêmica sob risco, muitas vezes se prefere a cirurgia. Quanto a pacientes com lesões multiarteriais, os resultados expostos abaixo, enquanto não se tem as informações do estudo FREEDOM, igualmente citado a seguir, favorecem a escolha do tratamento cirúrgico. Os quadros a seguir resumem de forma simplificada as indicações de revascularização, conforme as diretrizes da Sociedade Brasileira de Cardiologia.

Situação particular existe nos pacientes diabéticos que são candidatos a transplante renal, onde há indicação mais forte para cirurgia de revascula-

Tabela 1. Classes de evidências comparativas entre angioplastia coronariana e tratamento cirúrgico.

\section{PACIENTES COM LESÃO EM UM VASO (UNIARTERIAIS)}

\section{Características do paciente}

Com sintomas, apesar do tratamento médico otimizado*

Assintomático, com grande área isquêmica ou com disfunção $V E^{* *}$

Obstrução proximal de artéria interventricular anterior (DA)

Assintomático, sem área isquêmica, sem disfunção do VE e sem obstrução proximal de DA

\begin{tabular}{cc} 
Cirurgia & Angioplastia \\
I/A & I/A \\
I/A & I/A \\
IIa/A & IIa/A \\
III/C & III/C \\
\hline
\end{tabular}

$\left.{ }^{*}\right)$ independentemente da presença de isquemia e/ou viabilidade miocárdica

(**) implica ter viabilidade miocárdica

Tabela 2. Classes e níveis de evidência comparativos entre angioplastia coronariana e tratamento cirúrgico.

\section{PACIENTES COM LESÃO EM DOIS VASOS (BIARTERIAIS)}

\section{Características do paciente}

Com obstrução proximal de DA independente da presença de isquemia

Com obstrução de DA na presença de isquemia e/ou de disfunção do VE*

Sem obstrução de DA, com grande área de isquemia e/ou de disfunção do VE*

Sem obstrução de DA, com moderada área de isquemia e/ou de disfunção do VE*

\section{Cirurgia}

I/B

I/A

I/B

$\mathrm{Ila} / \mathrm{B}$
Angioplastia

I/B

I/A

I/B

$\mathrm{Ila} / \mathrm{B}$

${ }^{*}$ ) implica ter viabilidade miocárdica

Tabela 3. Classes e níveis de evidência comparativos entre angioplastia coronariana e tratamento cirúrgico.

\begin{tabular}{lcc}
\hline & PACIENTES COM SITUAÇÕES ESPECIAIS & \\
Características do paciente & Cirurgia & Angioplastia \\
Tronco CE não-protegido $(>50 \%)$ & $\mathrm{I} / \mathrm{A}$ & $\mathrm{III} / \mathrm{C}$ \\
Tronco CE protegido $(>50 \%)$ & $\mathrm{Ila} / \mathrm{C}$ & $\mathrm{I} / \mathrm{C}$ \\
Tronco CE $(>50 \%)$ em pacientes sem possibilidade cirúrgica* & - & $\mathrm{Ila} / \mathrm{C}$ \\
Angina limitante/refratária** & $\mathrm{I} / \mathrm{C}$ & $\mathrm{I} / \mathrm{C}$ \\
Estenose $50 \%$ a $70 \%$ s/ isquemia e/ou disfunção VE* & $\mathrm{III} / \mathrm{B}$ & $\mathrm{III} / \mathrm{B}$ \\
& & \\
\hline
\end{tabular}

(*) DPOC/IRC/câncer/sem enxerto

$\left.{ }^{* *}\right)$ independente do $n^{\circ}$ de artérias 
rização. Aproximadamente 20 a 30\% destes pacientes têm doença arterial coronariana significativa, que pode ser assintomática e não associada aos fatores de risco usuais. Estudo angiográfico de rotina evidenciou presença de lesões coronarianas significativas em $36 \%$ de pacientes diabéticos e em diálise, independentemente de sintomas. Portanto, diagnóstico invasivo ou não-invasivo para doença coronariana deve ser buscado em pacientes antes de submetêlos a transplante renal, e deve ser indicada revascularização se necessária $(8)$.

\section{RISCO CIRÚRGICO}

O controle peri-operatório do diabético demanda cuidados especiais. Entretanto, o DM não parece estar relacionado a maior risco de óbito imediato. Critérios de estimativa de risco, como o Sistema Europeu de Avaliação de Risco Cirúrgico (EuroSCORE) (10), não identifica sua presença como fator de risco imediato para cirurgia cardíaca. Estudo realizado no Instituto de Cardiologia do RS, com 1.629 pacientes operados no período de $1996 \mathrm{e}$ 1998, dos quais 24\% eram diabéticos, encontrou significativa maior freqüência de DM no gênero feminino ( $29 \%$ versus $22 \%, \mathrm{p}=0,006)$. No entanto, a análise multivariada não encontrou associação isolada de diabetes com risco para mortalidade peri-operatória. (11). A presença de DM e a necessidade de seu controle pós-operatório podem prolongar o tempo de internação em CTI ou hospitalar (12). Entretanto, na nossa experiência, este não tem sido um fator de prolongamento da internação.

O impacto de DM nos desfechos cirúrgicos foi analisado detalhadamente em uma série de $16.184 \mathrm{pa}^{-}$ cientes submetidos à revascularização cirúrgica do miocárdio, com e sem circulação extracorpórea. Comparativamente ao grupo não-diabético, o grupo $\mathrm{DM}$ era mais idoso e com menor fração de ejeção ventricular. DM foi identificado como fator independente de risco para prolongamento da internação em CTI, instabilidade e/ou infecção do esterno, insuficiência respiratória, delirium pós-operatório, acidente vascular cerebral, disfunção renal e reintubação para ventilação prolongada (13).

Em estudo retrospectivo, comparando populações homogêneas de pacientes submetidos à cirurgia de revascularização miocárdica em que a única variável foi a presença ou não de $\mathrm{DM}$, não houve diferença $\mathrm{em}$ mortalidade, mas ocorreram 3,5 vezes mais complicações neurológicas e 5 vezes mais complicações renais, além de 2 vezes maior índice de reoperações, de tempo de internação em CTI e de maior necessidade de transfusões (14).

Disfunção cognitiva é um problema bem conhecido no período peri-operatório de cirurgia cardíaca. Há sugestões de desfechos cognitivos piores no paciente diabético submetido à circulação extracorpórea (15).

A infecção, tanto superficial do esterno, do local de retirada da veia safena e a mais temida mediastinite, é complicação de comprovada maior incidência em DM. Em estudo recente, avaliando fatores de risco para freqüência de infecções pós-operatórias, encontrou-se que infecções por bactérias Gram-positivas são significativamente mais freqüentes nos diabéticos e as infecções por Gram-negativos são 2 vezes mais freqüentes na presença de diabetes mellitus (16).

É de fundamental importância buscar reconhecer a presença de DM em candidatos à cirurgia. A prevalência e os riscos de $\mathrm{DM}$ não-diagnosticado $\mathrm{em}$ pacientes submetidos à cirurgia de revascularização miocárdica foi analisada em uma série de 7.310 pacientes consecutivos. A prevalência de DM diagnosticada foi $29,6 \%$ e a prevalência de $\mathrm{DM}$ não-diagnosticada foi 5,2\% (glicemia igual ou maior que 126 $\mathrm{mg} / \mathrm{dl}$ ). Em DM não-diagnosticada, foram mais freqüentes a ressuscitação e reintubação traqueal, bem como o tempo de ventilação mecânica foi mais prolongado. A mortalidade peri-operatória foi significativamente maior neste grupo $(\mathrm{p}<0,01)(17)$.

\section{CONTROLE GLICÊMICO}

O controle deficiente da glicemia peri-operatória está relacionado a piores desfechos hospitalares, não apenas no paciente diabético, mas também no não-diabético. $\mathrm{O}$ estresse induzido pelo procedimento e pela circulação extra-corpórea pode levar a importante hiperglicemia. Níveis de glicemia maior que $150 \mathrm{mg} / \mathrm{dl}$ no período operatório e superior a $350 \mathrm{mg} / \mathrm{dl}$ durante a circulação extra-corpórea foram relacionados a aumento das complicações cardiovasculares, respiratórias, infecciosas, neurológicas e renais. Pacientes com deficiente controle glicêmico intraoperatório podem apresentar 7 vezes maior chance de complicações em relação aos demais (11).

\section{RESULTADOS}

A cirurgia de revascularização miocárdica em pacientes idosos (mais de 65 anos) e com diabetes resulta em redução significativa da mortalidade cardiovascular em geral, podendo alcançar redução de $44 \%$, como relatado no estudo CASS, comparável ao benefício da população não-diabética (18).

O DM, entretanto, atua como fator detrimental reconhecido para redução do benefício da revascu- 
larização em longo prazo. A mortalidade em período de 2 anos pós-operatórios, para pacientes de todas as idades, pode ser 2 vezes maior pela presença de DM (19). Estudos mais recentes confirmam o aumento progressivo e contínuo da mortalidade tardia devido à presença de DM, que chega ao dobro da mortalidade do restante da população tratada em acompanhamento de 9,5 anos. Outros fatores de risco avaliados não tiveram este comportamento (20). A despeito do comprometimento tardio causado por DM, o tratamento cirúrgico do paciente multiarterial com diabetes está associado à significativa melhora da sobrevida em relação àqueles submetidos a tratamento medicamentoso e à angioplastia percutânea por cateter, como relatado no estudo BARI (21). A melhor sobrevida neste estudo (mortalidade de $5,8 \%$ no grupo cirúrgico comparada com $20,6 \%$ no grupo percutâneo, no acompanhamento médio de 5,4 anos) esteve relacionada ao implante de pelo menos uma artéria mamária, ressaltando a importância deste tipo de enxerto na melhora do prognóstico tardio. Sabe-se que o implante de artéria mamária interna esquerda na artéria coronária descendente anterior se constitui em fator independente de melhora da sobrevida em longo prazo. Este benefício poderia ser estendido para o implante de 2 enxertos de artéria mamária, ou seja, um segundo enxerto, da artéria mamária interna direita para outra artéria coronária. Entretanto, não foi encontrado benefício significativo para sobrevida a longo prazo de implante bilateral em pacientes diabéticos. Em um estudo, esta possível maior longevidade foi encontrada nos pacientes da faixa etária entre 60 e 69 anos, enquanto acima dos 79 anos o benefício foi maior no grupo de apenas um implante unilateral (22).

Portanto, embora os riscos sejam maiores no paciente com diabetes, a cirurgia de revascularização, quando indicada, pode resultar em melhor sobrevida comparada ao tratamento clínico ou à angioplastia percutânea. $\mathrm{O}$ acompanhamento em período mais longo de grandes estudos randomizados, por 7 ou 8 anos, como o já referido BARI e o estudo EAST (23), demonstra a maior sobrevida de pacientes diabéticos revascularizados cirurgicamente, em relação à angioplastia, especialmente naqueles usuários de insulina ou com implantes de artéria mamária interna (24).

Por outro lado, estudos em pacientes multiarteriais comparando as três estratégias, ou seja, tratamento clínico, cirurgia ou angioplastia, como o estudo MASS-II (25), não encontraram diferença de prognóstico em relação à presença ou ausência de diabetes, embora tenham demonstrado significativa superioridade do tratamento cirúrgico na eliminação de sintomas de angina, na redução de eventos cardiovasculares e na redução do número de novas intervenções. Neste estudo, havia $18,8 \%$ de pacientes diabéticos. Após 1 ano de acompanhamento, $8,3 \%$ dos tratados clinicamente e $13,3 \%$ dos submetidos a angioplastia haviam sido submetidos a novas intervenções, comparado com apenas $0,5 \%$ do grupo cirúrgico.

Os estudos multicêntricos comparativos entre tratamento percutâneo com cateter e cirurgia são anteriores ao advento dos stents farmacológicos, que estão sendo introduzidos na prática intervencionista, na expectativa de apresentarem menores índices de reestenose. Os resultados em longo prazo, entretanto, não são conhecidos e estão ainda em avaliação. Está em andamento, promovido pelo National Heart, Lung and Blood Institute (NHLBI), dos Estados Unidos, o estudo FREEDOM (Future Revascularization Evaluation in Patients With Diabetes Mellitus: Optimal Management of Multivessel Disease), que tem o propósito de comparar a mortalidade em 5 anos de pacientes diabéticos que vão à cirurgia de revascularização ou a implante percutâneo de stents farmacológicos intracoronarianos. Esse estudo foi iniciado em abril de 2004 e ainda está recrutando pacientes. Outros estudos - BARI 2D e CarDIA - têm a mesma finalidade (http://clinicaltrials.gov/ct/gui/ show/NCT00086450).

\section{PATÊNCIA DOS ENXERTOS}

Avaliação sobre a patência de enxertos de veia safena e de artéria mamária interna (26), embasada em dados de 50.278 pacientes operados entre 1972 e 1999 , encontrou significativa maior patência para os implantes de artéria mamária em todas as situações, exceto quando implantadas na artéria coronária direita com lesões inferiores a $70 \%$ da luz do vaso. Os índices gerais não-ajustados, para 1, 5 e 10 anos p.o., foram de $93 \%, 88 \%$ e $90 \%$ de patência para artéria mamária e de $78 \%, 65 \%$ e $57 \%$ para veias safenas. Diabetes esteve associado a maior oclusão de enxertos $(\mathrm{p}=0,03)$, especialmente quando presente no gênero feminino $(\mathrm{p}=$ $0,002)$. Sabe-se que as oclusões de enxertos estão relacionadas ao diâmetro dos vasos, e que estes são mais finos em diabéticos, em mulheres e em pacientes de pequena estatura. Esta poderia ser a explicação para os achados deste estudo, bem como para aqueles encontrados na experiência do Instituto de Cardiologia do RS (11), como já relatado acima.

Em publicação mais recente e voltada para avaliar a influência das características dos pacientes na necessidade de reoperações coronarianas, o grupo acima citado (Cleveland Clinic), estudou uma série inicial de 48.758 
casos acompanhados a intervalos de 5 anos, por até $\mathbf{2 5}$ anos. A proporção de pacientes livres de reoperação foi de $99,6 \%, 98,4 \%, 93 \%, 82 \%, 72 \%$ e $65 \%$, respectivamente aos $1,5,10,15,20$ e 25 anos. As variáveis independentes que aumentaram a necessidade de reoperações foram idade menor na época da cirurgia, hipercolesterolemia, hipertrigliceridemia hipolipoproteinemia de baixa densidade, diabetes mellitus $(<0,0001)$ e doença arterial coronariana mais extensa (27). Portanto, DM está associado comprovadamente a maiores índices de oclusão de enxertos a médio e longo prazos, bem como a maior necessidade de reintervenções tardias para revascularização.

\section{CONCLUSÕES}

Quando comparados aos pacientes sem DM, os portadores de DM encaminhados à revascularização do miocárdio são mais idosos, apresentam doença arterial difusa, mais co-morbidades e pior prognóstico em longo prazo. As indicações de cirurgia seriam comprometimento uni, bi ou triarterial com envolvimento proximal da artéria DA, ou com disfunção ventricular esquerda, principalmente na presença de uma ou mais oclusões totais crônicas. O implante da artéria mamária interna está associado à melhora do prognóstico e diminuição da mortalidade. Ao indicar cirurgia de revascularização miocárdica no paciente diabético, devemos esperar melhoria significativa na sintomatologia, na qualidade de vida e na sobrevida em longo prazo, embora estes parâmetros sejam inferiores, no diabético, em comparação à população geral.

\section{REFERÊNCIAS}

1. Kirklin JW, Barratt-Boyes BG. Stenotic arteriosclerotic coronary artery disease. In: Kirklin JW, Barratt-Boyes BG (eds). Cardiac Surgery. $2^{a}$ ed. New York: Churchill Livingstone, 1993. p. $285-363$.

2. Schaan BD, Harzheim E, Gus I. Cardiac risk profile in diabetes mellitus and impaired fasting glycemia. Rev Saúde Pública 2004;38:529-36.

3. Sociedade Brasileira de Cardiologia. Diretrizes de Doença Coronariana Crônica - Angina Estável. Arq Bras Cardiol 2004;83(supl.II):1-43.

4. Liao L, Kong DF, Shaw LK, Sketch MH Jr, Milano CA, Lee KL, et al. A new anatomic score for prognosis after cardiac catheterization in patients with previous bypass surgery. J Am Coll Cardiol 2005;46:1684-92.

5. Thourani VH, Weintraub WS, Stein B, Gebhart SS, Craver JM, Jones EL, et al. Influence of diabetes mellitus on early and late outcome after coronary artery bypass grafting. Ann Thorac Surg 1999;67:1045-52.

6. Quadros A, Kalil R, Hanna F, Sarmento-Leite R, Gottschall CAM. Revascularização miocárdica nos pacientes com cardiopatia isquêmica e diabetes mellitus. Rev Soc Cardiol RS 2006; (no prelo)

7. Aronson D, Rayfield EJ. Diabetes and obesity. In: Fuster V R, Topol EJ (ed). Atherosclerosis and Coronary Artery Disease. Philadelphia: Lippincott-Raven, 1996.
8. Eagle KA, Guyton RA. ACC/AHA practice guidelines. Guideline update for coronary artery bypass graft surgery. Circulation 2004;110:e340-437.

9. Sociedade Brasileira de Cardiologia. Diretriz da cirurgia de revascularização miocárdica. Disponível em: <http:// publicacoescardiolbr/consenso/2004/ site/035pdf160 2004:1-60>.

10. Nashef S, Roques F, Michel P, Gauducheau E, Lemeshow S, Salamon S. The EuroSCORE Study group. European System for cardiac operative risk evaluation (EuroSCORE). Eur J Cardiothorac Surg 1999;16:9-13.

11. Behr PEB. O sexo feminino como fator de risco para a mortalidade hospitalar após a cirurgia de revascularização miocárdica (dissertação). Porto Alegre: Instituto de Cardiologia do Rio Grande do Sul / Fundação Universitária de Cardiologia, 2001.

12. Bucerius J, Gummert JF, Walther T, Doll N, Falk V, Schmitt DV, et al. Predictors of prolonged ICU stay after on-pump versus off-pump coronary bypass grafting. Intens Care Med 2004;30:88-95.

13. Bucerius J, Gummert JF, Walther T, Doll N, Falk V, Onnasch $\mathrm{JF}$, et al. Impact of diabetes mellitus on cardiac surgery outcome. Thorac Cardiovasc Surg 2003;51(1):11-6.

14. Morricone L, Ranucci M, Denti S, Cazzaniga A, Isgro G, Enrini $R$, et al. Diabetes and complications after cardiac surgery: comparison with a non-diabetic population. Acta Diabetol 1999;36(1-2):77-84.

15. Notzold A, Michel K, Khattab AA, Sievers HH, Huppe M. Diabetes mellitus increases adverse neurocognitive outcome after coronary artery bypass grafting surgery. Thorac Cardiovasc Surg 2006;54(5):307-12.

16. Garey KW, Kumar N, Dao T, Tam VH, Gentry LO. Risk factors for postoperative chest wound infections due to gram-negative bacteria in cardiac surgery patients. J Chemother 2006;18(4):402-8.

17. Lauruschkat AH, Arnrich B, Albert AA, Walter JA, Amann B, Rosendahl UP, et al. Prevalence and risks of undiagnosed diabetes mellitus in patients undergoing coronary artery bypass grafting. Circulation 2005;112(16):2397-402.

18. Barzilay JL, Kronmal RA, Bittner V, Eaker E, Evans C, Foster ED. Coronary artery disease and coronary artery bypass grafting in diabetic patients aged 65 years or more: a report from the Coronary Artery Surgery Study (CASS) Registry. Am J Cardiol 1994;74:334-9.

19. Herlitz J, Wognsen GB, Emanuelsson H, Haglid M, Karlson BW, Karlsson T, et al. Mortality and morbidity in diabetic and nondiabetic patients during a 2-year period after coronary artery bypass grafting. Diabetes Care 1996;19:698703.

20. Gao D, Grunwald GK, Rumsfeld JS, Schooley L, MacKenzie $T$, Shroyer AL. Time-varying risk factors for long-term mortality after coronary artery bypass graft surgery. Ann Thorac Surg 2006;81(3):793-9.

21. The BARI Investigators. Influence of diabetes on 5-year mortality and morbidity in a randomized trial comparing CABG and PTCA in patients with multivessel disease. Circulation 199;96:1761-9.

22. Toumpoulis IK, Anagnostopoulos CE, Balaram S, Swistel DG, Ashton RC Jr, DeRose JJ Jr. Does bilateral internal thoracic artery grafting increase long-term survival of diabetic patients? Ann Thorac Surg 2006;81:599-606.

23. King SB, Kosinski AS, Guyton RA, Lembo NJ, Weintraub WS. Eight-year mortality in the Emory Angioplasty versus Surgery Trial (EAST). J Am Coll Cardiol 2000;35:1116-21.

24. Brooks MM, Jones RH, Bach RG, Chaitman BR, Kern MJ, Orszulak TA, et al. The BARI Investigators. Predictors of mortality and mortality from cardiac causes in the Bypass Angioplasty Revascularization Investigation (BARI) randomized trial and registry. Circulation 2000;101:2682.

25. Hueb W, Soares P, Gersh BJ, Cesar LA, Luz PL, Puiq LB, et al. The Medicine, Angioplasty, or Surgery Study (MASS-II): A randomized, controlled clinical trial of three therapeutic strategies for multivessel coronary artery disease. One-year results. J Am Coll Cardiol 2004;43:1743-51. 
26. Sabik J, Lytle B, Blackstone $\mathrm{E}$, Houghtaling PL, Delos M, Cosgrove DM. Comparison of saphenous vein and internal thoracic artery graft patency by coronary system. Ann Thorac Surg 2005;79:544-51.

27. Sabik JF III, Blackstone EH, Gillinov AM, Banbury MK, Smedira NG, Lytle BW. Influence of patient characteristics and arterial grafts on freedom from coronary reoperation. $\mathbf{J}$ Thorac Cardiovasc Surg 2006;131:90-8.
Endereço para correspondência:

Renato A.K. Kalil

Unidade de Pesquisa do IC/FUC

Av. Princesa Isabel 370

90620-001 Porto Alegre, RS

Fax: (51) 3219-2802 ext.: 23, 24

E-mail: kalil@cardiologia.org.br/

editoracao.pc@cardiologia.org.br 